\title{
OPEN Ensemble machine learning prediction and variable importance analysis of 5-year mortality after cardiac valve and CABG operations
}

José Castela Forte $\mathbb{1}^{1,2,7 凶}$, Hubert E. Mungroop ${ }^{2}$, Fred de Geus², Maureen L. van der Grinten ${ }^{7}$, Hjalmar R. Bouma ${ }^{1,3}$, Ville Pettilä ${ }^{4}$, Thomas W. L. Scheeren (1) ${ }^{2}$, Maarten W. N. Nijsten ${ }^{5}$, Massimo A. Mariani ${ }^{6}$, Iwan C. C. van der Horst $\mathbb{1}^{5,8}$, Robert H. Henning ${ }^{1}$, Marco A. Wiering ${ }^{7}$ \& Anne H. Epema ${ }^{2}$

Despite having a similar post-operative complication profile, cardiac valve operations are associated with a higher mortality rate compared to coronary artery bypass grafting (CABG) operations. For long-term mortality, few predictors are known. In this study, we applied an ensemble machine learning (ML) algorithm to 88 routinely collected peri-operative variables to predict 5 -year mortality after different types of cardiac operations. The Super Learner algorithm was trained using prospectively collected peri-operative data from 8241 patients who underwent cardiac valve, CABG and combined operations. Model performance and calibration were determined for all models, and variable importance analysis was conducted for all peri-operative parameters. Results showed that the predictive accuracy was the highest for solitary mitral $(0.846[95 \% \mathrm{Cl} 0.812-0.880])$ and solitary aortic ( 0.838 [0.813-0.864]) valve operations, confirming that ensemble ML using routine data collected perioperatively can predict 5 -year mortality after cardiac operations with high accuracy. Additionally, post-operative urea was identified as a novel and strong predictor of mortality for several types of operation, having a seemingly additive effect to better known risk factors such as age and postoperative creatinine.

Whereas complications after cardiac operations are associated with increased risk of in-hospital mortality, only few predict long-term mortality. The best documented is post-operative acute kidney injury (AKI), a highly prevalent complication occurring in $15-30 \%$ of patients ${ }^{1,2}$ which is associated with both increased short- and long-term mortality ${ }^{1-4}$. The relation between postoperative AKI and mortality varies greatly per type of cardiac operation. Mortality risks related to AKI are well characterized for coronary artery bypass grafting (CABG), but less well studied in valve operations, despite these accounting for $24 \%$ of all cardiac operations and having higher mortality rates ${ }^{5,6}$. Recently, Bouma et al..$^{5}$, showed post-operative AKI to be strongly associated with an increase in long-term mortality in patients with solitary valve and combined valve and CABG operations. Remarkably, even a mild impairment in renal function well below the threshold for AKI-1 (i.e., a mere 10\% post-operative increase

\footnotetext{
${ }^{1}$ Department of Clinical Pharmacy and Pharmacology, University of Groningen, University Medical Center Groningen, Hanzeplein 1, P.O. Box 30.001, 9700 RB Groningen, The Netherlands. ${ }^{2}$ Department of Anesthesiology, University of Groningen, University Medical Center Groningen, Groningen, The Netherlands. ${ }^{3}$ Department of Internal Medicine, University of Groningen, University Medical Center Groningen, Groningen, The Netherlands. ${ }^{4}$ Division of Intensive Care Medicine, Department of Anesthesiology, Intensive Care and Pain Medicine, University of Helsinki and Helsinki University Hospital, Helsinki, Finland. ${ }^{5}$ Department of Critical Care, University of Groningen, University Medical Center Groningen, Groningen, The Netherlands. ${ }^{6}$ Department of Cardiothoracic Surgery, University of Groningen, University Medical Center Groningen, Groningen, The Netherlands. ${ }^{7}$ Bernoulli Institute for Mathematics, Computer Science and Artificial Intelligence, University of Groningen, Groningen, The Netherlands. ${ }^{8}$ Department of Intensive Care, Maastricht University Medical Centre+, University Maastricht, Maastricht, The Netherlands. ${ }^{\circledR}$ email: j.n.alves.castela.cardoso.forte@umcg.nl
} 
in serum creatinine) significantly increased long-term mortality risk in solitary valve operations ${ }^{5}$. Therefore, to date postoperative AKI represents the best studied organ injury related early marker of long-term mortality risk after cardiac operations.

Previously, we have demonstrated that machine learning (ML) predictive models proved superior to classical multivariable analysis in identifying patients at increased risk of long-term mortality after CABG operations ${ }^{7}$. Moreover, a unique property of ML is its ability to identify parameters predicting mortality and rank their importance by variable importance analysis. However, while ML analyses gain popularity in peri-operative care $^{8}$, studies using ML techniques for long-term mortality analysis after cardiac valve operations are lacking. Several studies in different fields of healthcare have shown ensemble ML algorithms to be more accurate than individual algorithms in modelling complex outcomes such as mortality in critically ill patients ${ }^{9}$ and mortality following cardiac arrest ${ }^{10}$. In anesthesiology, recent studies showed that different machine learning algorithms could accurately predict acute hypotensive episodes $10 \mathrm{~min}$ in advance using patient characteristics and physiological variables ${ }^{11-13}$.

In this study, we combined multiple ML algorithms into an ensemble using the Super Learner (SL) algorithm ${ }^{14}$. This ensemble ML algorithm was trained to predict 5-year mortality in a large prospective cohort of patients undergoing cardiac valve, $\mathrm{CABG}$, or combined operations using routinely collected peri-operative data in a single tertiary care hospital. We compared the accuracy of two SL training methodologies, using a targeted approach with patients split per operation type compared to the entire, unselected population. Furthermore, variable importance analysis was conducted to identify the strongest predictors of mortality.

\section{Results}

Patient characteristics and mortality per operation type. Patient characteristics, descriptives of all variables used in this study and mortality data per operation type are summarized in Table 1 (and Table 1 of the "Supplementary material"). Five years mortality rate of the full patient cohort was $16.5 \%$. Operations involving valve procedures showed higher mortality amounting $16.9 \%$ for aortic valve alone, $19.7 \%$ for mitral valve alone, $21.0 \%$ for combined aortic valve/CABG and $28.9 \%$ for combined mitral valve/CABG (Table 1 ). Accordingly, mortality rate for CABG-only $(13.8 \%)$ was lower than for the entire cohort.

Machine learning analysis. As a first step in the ML based prediction of long-term mortality, the ensemble was trained on the full cohort (SL1; Fig. 5, left part). ROC curves and their respective AUROCs were established for the full cohort and the different cardiac operation types (Fig. 1). SL1 achieved an AUROC of 0.810 [0.7980.823 . When analyzed per operation type, the accuracy of SL1 was highest for solitary mitral valve $(0.846)$ and solitary aortic valve operations (0.838), and lowest for solitary CABG (0.784) and mitral valve/CABG (0.796). In addition, the comparison between SL1 and the trained GLM showed that the SL1 significantly outperformed GLM (AUROC 0.756 [0.725-0.787]) for the full cohort ( $P=0.0016$; Fig. 1) as well as for solitary aortic valve and combined aortic valve and CABG $(P<0.01$; Table 2 in the "Supplementary material"). Thus, SL1 produced sound long-term mortality prediction based on peri-operative routinely collected patient and operation data.

Next, we performed a similar analysis based on SL training per operation type, by making five training sets using $80 \%$ of the relevant patients to train five weighted ensembles (SL2-SL6). Comparison of AUROCs between SL1 versus SL2-6, showed identical ranking for specific operation subgroups. Predictive performance between the models generated by SL1 compared those from SL2 to SL6 did not differ (Fig. 1; Table 2 in the "Supplementary material"). SL3 and SL4 also outperformed GLM $(P<0.01$; Table 4 in the "Supplementary material"). Lastly, because of its potential ability to identify patients at high risk prior to surgery, we examined the predictive performance when only pre-operative data are included. As expected, the model trained only on pre-operative data showed inferior performance to the full peri-operative model (AUROC 0.718 [0.687-0.749], $P<0.01$, Fig. 12 in the "Supplementary material").

Calibration, sensitivity analysis and adjusted risk thresholds based on predicted probability of mortality. Calibration of SL1 and SL2-6 was good for most models (Table 5 and Figs. 1-11 of the "Supplementary material"). Using the adjusted thresholds based on the Youden index and on a 50\% increased risk of mortality lead to improved model sensitivity and specificity (Fig. 2). For all operations, the thresholds based on the Youden index approximated the baseline absolute mortality risk. Compared to the default threshold of 50\% mortality risk, both the thresholds based on the Youden index and the thresholds defined by a 50\% increased risk of mortality increased sensitivity substantially for all types of operation (Tables 6-15 of the "Supplementary material"). For the Youden index thresholds, this was paired with a steeper decrease in specificity than for the thresholds at $50 \%$ increased risk of mortality. As Table 2 shows, the threshold representing $50 \%$ increase in risk improved the number of patients correctly classified as "non-survivor" for all types of operation. The largest increase in correctly classified "non-survivors" was observed for aortic valve, CABG, combined aortic valve and $\mathrm{CABG}$, and for all operations combined (3-, 4.7-, 2.2-, and 3-fold increase).

Variable importance analysis. Unexpectedly, variable importance analysis of all operations combined $(n=8142)$ revealed serum urea at day 4 after operation as the top predictor variable for 5-year mortality (Fig. 3). Serum urea was also found the top predictor in all operation types, except for the smallest group $(\mathrm{n}=367)$, combined mitral valve and CABG operations. Other important predictive variables included patient age, serum urea at other time points, indicators of kidney function, and serum markers for organ damage and inflammation. To better illustrate the impact of the changes in these variable and possible interactions, we constructed probability plots of the two highest ranking variables in all patients (Fig. 4). Mortality risk steeply increased from day 4 urea levels of $10 \mathrm{mmol} / \mathrm{L}$, reaching a plateau at $30 \mathrm{mmol} / \mathrm{L}$ denoting a $50 \%$ increase in absolute risk compared 


\begin{tabular}{|c|c|c|c|c|c|c|}
\hline & \multirow{2}{*}{\begin{tabular}{|l|} 
CABG \\
$\mathrm{N}=4514$ \\
\end{tabular}} & \multirow{2}{*}{\begin{tabular}{|l|} 
Aortic valve \\
$\mathrm{N}=1663$
\end{tabular}} & \multirow{2}{*}{\begin{tabular}{|l|} 
Mitral valve \\
$\mathrm{N}=\mathbf{8 8 4}$ \\
\end{tabular}} & \multirow{2}{*}{\begin{tabular}{|l|} 
Aortic + coronary \\
$\mathrm{N}=813$ \\
\end{tabular}} & \multirow{2}{*}{\begin{tabular}{|l|} 
Mitral + coronary \\
$\mathrm{N}=367$ \\
\end{tabular}} & \multirow[b]{2}{*}{ P value } \\
\hline & & & & & & \\
\hline BMI & 27.34 & 27.01 & 26.03 & 27.40 & 26.56 & $<0.001$ \\
\hline Gender & & & & & & $<0.001$ \\
\hline Female & $1028(22.77 \%)$ & $676(40.65 \%)$ & $424(47.96 \%)$ & $285(35.06 \%)$ & $125(34.06 \%)$ & \\
\hline Male & $3486(77.23 \%)$ & $987(59.35 \%)$ & $460(52.04 \%)$ & $528(64.94 \%)$ & $242(65.94 \%)$ & \\
\hline Age & 66.26 & $64.80(13.79)$ & $62.62(13.62)$ & $72.25(8.39)$ & $69.28(8.57)$ & $<0.001$ \\
\hline Pre-operative eCCR & 71.42 & $72.32(21.84)$ & 74.83 & 74.54 & 65.78 & 0.065 \\
\hline Post-operative eCCR & 66.99 & $70.50(39.87)$ & $67.43(27.26)$ & $62.94(24.22)$ & $59.03(23.90)$ & $<0.001$ \\
\hline Per-operative eCCR decrease & 4.44 & $1.82(32.85)$ & $7.40(95.76)$ & $11.60(106.61)$ & $6.75(16.49)$ & 0.001 \\
\hline Pre-operative eCCR ratio & 1.13 & $1.11(0.38)$ & $1.27(2.26)$ & $1.25(1.18)$ & $1.23(0.50)$ & 0.001 \\
\hline $\begin{array}{l}\text { Creatinine within } 24 \mathrm{~h} \text { before } \\
\text { surgery }(\mu \mathrm{mol} / \mathrm{L})\end{array}$ & 102.69 & $100.31(78.26)$ & 98.75 (44.46) & $104.65(79.01)$ & $107.90(72.40)$ & 0.138 \\
\hline Pre-operative creatinine & 101.95 & $99.21(72.06)$ & $98.15(40.11)$ & $102.89(68.31)$ & $107.11(70.80)$ & 0.110 \\
\hline $\begin{array}{l}\text { Creatinine } 12-24 \mathrm{~h} \text { after } \\
\text { surgery }\end{array}$ & 91.83 & $89.10(74.79)$ & $89.15(45.57)$ & $96.46(68.71)$ & $102.09(65.22)$ & 0.002 \\
\hline Creatinine $24 \mathrm{~h}$ after surgery & 92.84 & $90.71(73.06)$ & $91.85(45.48)$ & $98.40(70.21)$ & $103.72(65.53)$ & 0.002 \\
\hline $\begin{array}{l}\text { Creatinine at day } 2 \text { after } \\
\text { surgery }\end{array}$ & 102.72 & $99.13(73.60)$ & $96.19(49.84)$ & $104.08(66.63)$ & $107.76(56.25)$ & 0.006 \\
\hline $\begin{array}{l}\text { Creatinine at day } 4 \text { after } \\
\text { surgery }\end{array}$ & 98.61 & $94.35(75.25)$ & $93.75(57.22)$ & $100.73(74.97)$ & $104.61(69.26)$ & 0.007 \\
\hline $\begin{array}{l}\text { Maximum post-operative } \\
\text { creatinine }\end{array}$ & 111.03 & $108.53(86.88)$ & $110.96(64.41)$ & $119.15(86.85)$ & $126.22(76.60)$ & $<0.001$ \\
\hline $\begin{array}{l}\text { Absolute difference in } \\
\text { creatinine }\end{array}$ & 9.08 & 9.32 & $12.81(46.89)$ & $16.26(43.28)$ & $19.11(44.45)$ & $<0.001$ \\
\hline $\begin{array}{l}\text { Relative difference in } \\
\text { creatinine }\end{array}$ & 1.10 & 1.09 & 1.19 & $1.19(0.79)$ & $1.19(0.40)$ & $<0.001$ \\
\hline $\begin{array}{l}\text { Percentual difference in } \\
\text { creatinine }\end{array}$ & 10.20 & 8.71 & 19.27 & $19.45(79.05)$ & $18.96(39.91)$ & $<0.001$ \\
\hline $\begin{array}{l}\text { Urea within } 24 \mathrm{~h} \text { before } \\
\text { surgery }(\mathrm{mmol} / \mathrm{L})\end{array}$ & 6.96 & 7.25 & 8.09 & $7.57(3.27)$ & $8.20(3.76)$ & $<0.001$ \\
\hline Pre-operative urea & $6.98(3.29)$ & $7.34(4.03)$ & 8.25 & $7.51(3.12)$ & $8.52(6.11)$ & $<0.001$ \\
\hline Urea $12-24 \mathrm{~h}$ after surgery & $7.24(5.47)$ & $8.11(16.73)$ & 8.55 & $8.19(9.95)$ & $9.37(8.95)$ & $<0.001$ \\
\hline Urea at day 2 after surgery & $10.13(23.15)$ & $10.51(25.40)$ & 11.89 & $14.51(35.13)$ & $12.76(24.00)$ & $<0.001$ \\
\hline Urea at day 4 after surgery & $8.49(24.49)$ & $9.32(29.78)$ & 10.74 & $14.14(49.26)$ & $11.35(16.44)$ & $<0.001$ \\
\hline Maximum CPB flow & $4.63(1.47)$ & $4.12(1.97)$ & $3.96(2.05)$ & $4.02(2.05)$ & $4.14(2.01)$ & $<0.001$ \\
\hline Duration of perfusion & $100.07(38.64)$ & $125.29(48.12)$ & $169.76(73.36)$ & $168.07(50.43)$ & $214.06(77.13)$ & 0.000 \\
\hline Aortic cross-clamp time & $58.94(25.42)$ & $83.36(32.28)$ & $109.93(53.19)$ & $110.25(31.48)$ & $137.22(52.95)$ & 0.080 \\
\hline HR at start surgery & $62.22(12.90)$ & $67.14(14.00)$ & $70.80(17.52)$ & $62.55(13.90)$ & $66.17(16.06)$ & 0.000 \\
\hline HR during perfusion & $66.39(57.83)$ & $61.24(55.15)$ & $61.80(53.20)$ & $60.09(57.94)$ & $63.58(61.49)$ & $<0.001$ \\
\hline SBP at start surgery $(\mathrm{mmHg})$ & $113.54(34.62)$ & $108.81(31.98)$ & $102.25(31.32)$ & $109.36(33.49)$ & $105.28(29.93)$ & 0.002 \\
\hline SBP during perfusion & $61.76(21.39)$ & $63.37(22.60)$ & $63.17(20.39)$ & $63.96(20.10)$ & $62.83(22.03)$ & $<0.001$ \\
\hline DBP at start surgery $(\mathrm{mmHg})$ & $64.81(31.81)$ & $61.87(29.18)$ & $60.45(27.90)$ & $60.51(29.65)$ & $58.87(22.32)$ & 0.012 \\
\hline DBP during perfusion & $56.53(18.09)$ & $58.52(18.88)$ & $57.58(17.00)$ & $59.16(17.66)$ & $57.39(17.37)$ & $<0.001$ \\
\hline CVP at start surgery $(\mathrm{mmHg})$ & $12.58(30.79)$ & $11.96(28.43)$ & $13.88(30.11)$ & $12.89(32.66)$ & $12.32(24.81)$ & $<0.001$ \\
\hline CVP during perfusion & $6.62(8.31)$ & $5.03(9.45)$ & $4.78(15.07)$ & $5.65(5.60)$ & $4.44(7.75)$ & 0.653 \\
\hline $\mathrm{PaCO}_{2}$ at start surgery $(\mathrm{kPa})$ & $5.02(0.63)$ & $5.08(0.70)$ & $5.03(0.69)$ & $5.07(0.64)$ & $5.01(0.72)$ & $<0.001$ \\
\hline $\mathrm{PaCO}_{2}$ during perfusion & $5.04(0.54)$ & $5.17(0.57)$ & $5.18(0.62)$ & $5.09(0.51)$ & $5.13(0.57)$ & 0.010 \\
\hline $\mathrm{PaCO}_{2}$ at end surgery & $4.84(0.59)$ & $4.87(0.63)$ & $4.99(0.74)$ & $4.89(0.62)$ & $5.04(0.72)$ & $<0.001$ \\
\hline $\mathrm{PaO}_{2}$ at start surgery $(\mathrm{kPa})$ & $21.49(14.95)$ & $22.11(14.43)$ & $22.03(14.74)$ & $20.65(12.85)$ & $19.81(12.93)$ & $<0.001$ \\
\hline $\mathrm{PaO}_{2}$ during perfusion & $26.70(10.88)$ & $25.59(10.36)$ & $25.88(9.82)$ & $25.87(9.37)$ & $26.82(10.52)$ & 0.018 \\
\hline $\mathrm{PaO}_{2}$ at end surgery & $17.79(11.58)$ & $22.27(13.04)$ & $21.93(12.82)$ & $21.30(12.62)$ & $20.37(11.37)$ & 0.001 \\
\hline $\mathrm{SaO}_{2}$ at start surgery (\%) & $0.98(0.03)$ & $0.98(0.03)$ & $0.98(0.05)$ & $0.98(0.03)$ & $0.98(0.02)$ & $<0.001$ \\
\hline $\mathrm{SaO}_{2}$ during perfusion & $0.99(0.03)$ & $0.99(0.05)$ & $0.99(0.05)$ & $0.99(0.03)$ & $0.99(0.06)$ & 0.206 \\
\hline $\mathrm{SaO}_{2}$ end surgery & $0.98(0.03)$ & $0.99(0.04)$ & $0.98(0.04)$ & $0.98(0.04)$ & $0.98(0.02)$ & 0.152 \\
\hline ICU stay (hours) & $52.44(163.21)$ & $47.51(138.81)$ & $88.79(216.86)$ & $88.72(260.41)$ & $141.13(267.53)$ & $<0.001$ \\
\hline $\begin{array}{l}\text { ESR within } 24 \mathrm{~h} \text { before } \\
\text { surgery }(\mathrm{mm} / \mathrm{h})\end{array}$ & $20.61(19.57)$ & $18.63(19.96)$ & $20.21(19.40)$ & $22.23(20.25)$ & $23.01(21.03)$ & $<0.001$ \\
\hline Pre-operative ESR & $20.85(19.77)$ & $17.98(19.27)$ & $19.22(19.06)$ & $21.22(19.82)$ & $23.15(19.74)$ & $<0.001$ \\
\hline $\begin{array}{l}\text { LDH within } 24 \mathrm{~h} \text { before } \\
\text { surgery (U/L) }\end{array}$ & $227.71(75.41)$ & $248.34(115.16)$ & $259.90(169.51)$ & $235.06(70.41)$ & $228.79(66.45)$ & $<0.001$ \\
\hline Continued & & & & & & \\
\hline
\end{tabular}




\begin{tabular}{|c|c|c|c|c|c|c|}
\hline & \multirow{2}{*}{\begin{tabular}{|l|} 
CABG \\
$\mathrm{N}=4514$ \\
\end{tabular}} & \multirow{2}{*}{\begin{tabular}{|l|} 
Aortic valve \\
$\mathrm{N}=1663$ \\
\end{tabular}} & \multirow{2}{*}{\begin{tabular}{|l|} 
Mitral valve \\
$\mathrm{N}=\mathbf{8 8 4}$ \\
\end{tabular}} & \multirow{2}{*}{\begin{tabular}{|l|} 
Aortic + coronary \\
$\mathrm{N}=813$ \\
\end{tabular}} & \multirow{2}{*}{\begin{tabular}{|l|} 
Mitral + coronary \\
$\mathrm{N}=367$ \\
\end{tabular}} & \multirow[b]{2}{*}{$P$ value } \\
\hline & & & & & & \\
\hline Pre-operative LDH & $228.65(76.10)$ & $250.27(142.33)$ & $273.05(428.61)$ & $236.90(74.54)$ & $230.82(74.38)$ & $<0.001$ \\
\hline LDH $12-24 \mathrm{~h}$ after surgery & $338.15(273.89)$ & $396.80(179.67)$ & $480.19(484.17)$ & $456.26(497.74)$ & $510.39(662.83)$ & $<0.001$ \\
\hline LDH at day 2 after surgery & $338.30(233.89)$ & $388.29(252.01)$ & $461.39(444.76)$ & $446.04(312.62)$ & 474.68 (264.37) & $<0.001$ \\
\hline LDH at day 4 after surgery & $327.78(882.49)$ & $382.96(703.42)$ & 413.65 (329.29) & $424.52(461.76)$ & $439.23(340.88)$ & $<0.001$ \\
\hline $\begin{array}{l}\text { Maximum post-operative } \\
\text { LDH }\end{array}$ & $421.61(896.25)$ & $461.39(377.02)$ & $568.32(731.73)$ & $558.72(709.25)$ & $592.21(543.21)$ & $<0.001$ \\
\hline $\begin{array}{l}\text { Blood glucose } 0-6 \mathrm{~h} \text { after } \\
\text { surgery }(\mathrm{mmol} / \mathrm{L})\end{array}$ & $9.41(2.46)$ & $8.41(2.48)$ & $8.47(2.84)$ & $8.48(2.73)$ & $9.02(2.70)$ & $<0.001$ \\
\hline $\begin{array}{l}\text { Blood glucose 6-12 h after } \\
\text { surgery }\end{array}$ & $10.22(2.43)$ & $9.56(2.00)$ & $9.49(2.27)$ & $9.67(2.15)$ & $9.57(2.26)$ & $<0.001$ \\
\hline $\begin{array}{l}\text { Blood glucose } 12-24 \mathrm{~h} \text { after } \\
\text { surgery }\end{array}$ & $9.14(2.48)$ & $8.39(2.07)$ & $8.17(2.21)$ & $8.27(2.13)$ & $8.12(2.08)$ & $<0.001$ \\
\hline $\begin{array}{l}\text { Maximum post-operative } \\
\text { glucose }\end{array}$ & $11.19(4.37)$ & $10.38(3.84)$ & $10.48(2.58)$ & $10.53(2.24)$ & $10.82(2.69)$ & $<0.001$ \\
\hline $\begin{array}{l}\text { Hb within } 24 \mathrm{~h} \text { before sur- } \\
\text { gery }(\mathrm{mmol} / \mathrm{L})\end{array}$ & $8.47(1.09)$ & $8.45(1.06)$ & $8.27(1.20)$ & $8.35(1.00)$ & $8.30(1.11)$ & $<0.001$ \\
\hline Pre-operative $\mathrm{Hb}$ & $8.19(1.36)$ & $8.24(1.78)$ & $8.10(1.65)$ & $8.26(2.44)$ & $8.34(2.90)$ & $<0.001$ \\
\hline $\mathrm{Hb} 0-6 \mathrm{~h}$ after surgery & $5.64(0.73)$ & $5.69(0.76)$ & $5.78(0.82)$ & $5.52(0.76)$ & $5.57(0.84)$ & 0.135 \\
\hline $\mathrm{Hb} 6-12 \mathrm{~h}$ after surgery & $6.02(0.85)$ & $6.35(1.16)$ & $6.22(0.93)$ & $5.92(0.84)$ & $5.80(0.89)$ & $<0.001$ \\
\hline $\mathrm{Hb} 12-24$ h after surgery & $6.18(0.78)$ & $6.40(0.85)$ & $6.25(0.88)$ & $6.01(0.77)$ & $5.92(0.80)$ & $<0.001$ \\
\hline $\mathrm{Hb}$ at day 2 after surgery & $6.31(0.78)$ & $6.26(0.81)$ & $6.09(0.86)$ & $6.01(0.75)$ & $5.92(0.76)$ & $<0.001$ \\
\hline $\mathrm{Hb}$ at day 4 after surgery & $6.52(0.87)$ & $6.40(1.33)$ & $6.22(0.89)$ & $6.07(0.81)$ & $5.97(0.82)$ & $<0.001$ \\
\hline Minimum post-operative $\mathrm{Hb}$ & $5.31(0.69)$ & $5.41(0.73)$ & $5.29(0.77)$ & $5.11(0.65)$ & $5.01(0.70)$ & $<0.001$ \\
\hline $\begin{array}{l}\text { Leukocytes within } 24 \mathrm{~h} \\
\text { before surgery }\left(\times 10^{9} / \mathrm{L}\right)\end{array}$ & $7.84(2.73)$ & $7.44(2.70)$ & $7.62(3.32)$ & $7.77(3.22)$ & $7.74(2.18)$ & $<0.001$ \\
\hline Pre-operative leukocytes & $8.01(2.96)$ & $7.53(2.63)$ & $7.79(3.12)$ & $7.88(2.99)$ & $7.88(2.46)$ & $<0.001$ \\
\hline $\begin{array}{l}\text { Leukocytes 12-24 h after } \\
\text { surgery }\end{array}$ & $13.95(4.41)$ & $13.71(4.36)$ & $13.79(4.17)$ & $13.57(4.93)$ & $13.49(4.22)$ & $<0.001$ \\
\hline $\begin{array}{l}\text { Leukocytes at day } 2 \text { after } \\
\text { surgery }\end{array}$ & $17.08(4.82)$ & $15.79(4.81)$ & $15.99(5.12)$ & $16.14(4.74)$ & $16.49(4.85)$ & 0.051 \\
\hline $\begin{array}{l}\text { Leukocytes at day } 4 \text { after } \\
\text { surgery }\end{array}$ & $11.52(4.15)$ & $10.00(4.06)$ & $10.93(9.74)$ & $10.99(3.94)$ & $11.96(4.78)$ & $<0.001$ \\
\hline $\begin{array}{l}\text { Thrombocytes within } 24 \mathrm{~h} \\
\text { before surgery }\left(\times 10^{9} / \mathrm{L}\right)\end{array}$ & $246.55(73.47)$ & $231.91(67.44)$ & $235.83(72.42)$ & $234.86(69.37)$ & $239.06(72.70)$ & $<0.001$ \\
\hline Pre-operative thrombocytes & $238.69(78.71)$ & $224.72(71.67)$ & $230.34(75.95)$ & $230.41(73.07)$ & $233.71(76.22)$ & $<0.001$ \\
\hline $\begin{array}{l}\text { Thrombocytes } 0-6 \mathrm{~h} \text { after } \\
\text { surgery }\end{array}$ & $152.85(52.79)$ & $131.80(44.10)$ & $132.02(44.78)$ & $129.11(46.63)$ & $131.97(48.12)$ & $<0.001$ \\
\hline $\begin{array}{l}\text { Thrombocytes } 6-12 \mathrm{~h} \text { after } \\
\text { surgery }\end{array}$ & $171.17(58.06)$ & $149.14(49.54)$ & $141.39(48.69)$ & $136.37(47.14)$ & $140.20(54.93)$ & $<0.001$ \\
\hline $\begin{array}{l}\text { Thrombocytes } 12-24 \mathrm{~h} \text { after } \\
\text { surgery }\end{array}$ & $174.48(57.73)$ & $151.22(50.74)$ & $141.85(47.48)$ & $136.92(46.77)$ & $138.73(53.92)$ & $<0.001$ \\
\hline $\begin{array}{l}\text { ALAT within } 24 \mathrm{~h} \text { before } \\
\text { surgery (U/L) }\end{array}$ & $40.56(35.46)$ & $28.54(26.10)$ & $31.48(29.11)$ & $30.31(28.08)$ & $31.99(26.70)$ & $<0.001$ \\
\hline Pre-operative ALAT & $40.80(35.28)$ & $28.86(27.93)$ & $33.58(57.80)$ & $30.40(26.64)$ & $33.25(34.18)$ & $<0.001$ \\
\hline ALAT $12-24 \mathrm{~h}$ after surgery & $37.49(79.01)$ & $29.24(37.05)$ & $43.72(160.67)$ & $35.59(135.12)$ & $46.14(198.66)$ & $<0.001$ \\
\hline ALAT at day 2 after surgery & $37.57(146.05)$ & $31.20(88.67)$ & $44.26(123.93)$ & $40.56(168.31)$ & $40.43(105.37)$ & 0.002 \\
\hline $\begin{array}{l}\text { ASAT within } 24 \mathrm{~h} \text { before } \\
\text { surgery (U/L) }\end{array}$ & $32.72(20.36)$ & $29.50(20.19)$ & $31.83(24.10)$ & $28.95(15.01)$ & $31.08(28.86)$ & 0.169 \\
\hline Pre-operative ASAT & $33.18(24.15)$ & $30.11(24.14)$ & $37.82(166.12)$ & $29.45(15.34)$ & $30.90(18.47)$ & $<0.001$ \\
\hline ASAT $12-24 \mathrm{~h}$ after surgery & $59.82(108.39)$ & $71.96(83.88)$ & $112.66(241.82)$ & $98.08(206.81)$ & $121.96(283.96)$ & $<0.001$ \\
\hline ASAT at day 2 after surgery & $53.36(171.29)$ & $58.70(113.12)$ & $92.51(194.80)$ & $89.26(347.04)$ & $90.90(115.64)$ & 0.011 \\
\hline ASAT at day 4 after surgery & $55.12(422.01)$ & $54.44(197.38)$ & $68.11(217.54)$ & $72.37(435.80)$ & $71.26(248.91)$ & $<0.001$ \\
\hline $\begin{array}{l}\text { Neutrophils } 12-24 \mathrm{~h} \text { after } \\
\text { surgery }\left(\times 10^{9} / \mathrm{L}\right)\end{array}$ & $12.29(3.86)$ & $12.07(3.86)$ & $12.07(3.80)$ & $11.86(3.96)$ & $11.79(3.82)$ & 0.584 \\
\hline $\begin{array}{l}\text { Monocytes } 12-24 \mathrm{~h} \text { after } \\
\text { surgery }\left(\times 10^{9} / \mathrm{L}\right)\end{array}$ & $1.10(1.73)$ & $1.32(2.07)$ & $1.51(2.25)$ & $1.42(2.33)$ & $1.34(2.20)$ & 0.004 \\
\hline $\begin{array}{l}\text { Lymphocytes } 12-24 \mathrm{~h} \text { after } \\
\text { surgery }\left(\times 10^{9} / \mathrm{L}\right)\end{array}$ & $1.05(2.05)$ & $1.12(1.86)$ & $1.35(2.60)$ & $1.15(1.93)$ & $1.34(3.11)$ & $<0.001$ \\
\hline 5-year mortality: & & & & & & 0.001 \\
\hline Alive & $3890(86.18 \%)$ & $1382(83.10 \%)$ & $710(80.32 \%)$ & $642(78.97 \%)$ & $261(71.12 \%)$ & $<0.001$ \\
\hline Deceased & $624(13.82 \%)$ & $281(16.90 \%)$ & $174(19.68 \%)$ & $171(21.03 \%)$ & $106(28.88 \%)$ & \\
\hline Minimum body temperature & $31.71(1.82)$ & $31.20(2.60)$ & $30.76(2.36)$ & $31.23(2.17)$ & $30.89(1.86)$ & \\
\hline Continued & & & & & & \\
\hline
\end{tabular}




\begin{tabular}{|l|l|l|l|l|l|l|}
\hline & CABG & Aortic valve & Mitral valve & Aortic + coronary & Mitral + coronary & \multirow{2}{*}{ P value } \\
\cline { 2 - 6 } & $\mathbf{N = 4 5 1 4}$ & $\mathbf{N = 1 6 6 3}$ & $\mathbf{N}=\mathbf{8 8 4}$ & $\mathbf{N}=\mathbf{8 1 3}$ & $\mathbf{N}=\mathbf{3 6 7}$ & $<0.001$ \\
\hline AKI staging & & & & & & $<0.001^{*}$ \\
\hline No AKI & $3063(67.86 \%)$ & $1142(68.67 \%)$ & $584(66.06 \%)$ & $462(56.83 \%)$ & $199(54.22 \%)$ & \\
\hline Mild subclinical AKI & $841(18.63 \%)$ & $268(16.12 \%)$ & $133(15.05 \%)$ & $145(17.84 \%)$ & $62(16.89 \%)$ & \\
\hline Moderate subclinical AKI & $142(3.15 \%)$ & $51(3.07 \%)$ & $26(2.94 \%)$ & $37(4.55 \%)$ & $14(3.81 \%)$ & \\
\hline AKI 1-3 & $468(10.37 \%)$ & $202(12.15 \%)$ & $141(15.95 \%)$ & $169(20.79 \%)$ & $92(25.07 \%)$ & \\
\hline AKI 1 & $441(9.77 \%)$ & $191(11.49 \%)$ & $126(14.25 \%)$ & $157(19.31 \%)$ & $90(24.52 \%)$ & \\
\hline AKI 2 & $9(0.20 \%)$ & $6(0.36 \%)$ & $11(1.24 \%)$ & $6(0.74 \%)$ & $2(0.54 \%)$ & \\
\hline AKI 3 & $18(0.40 \%)$ & $5(0.30 \%)$ & $4(0.45 \%)$ & $6(0.74 \%)$ & $0(0 \%)$ & \\
\hline
\end{tabular}

Table 1. Descriptives table per operation type. All values presented as mean (95\% CI), and categorical variable with the percentage in parentheses. $B M I$ body mass index, $e C C R$ estimated creatinine clearance, $C P B$ cardiopulmonary bypass, $H R$ heart rate, $S B P$ systolic blood pressure, $D B P$ diastolic blood pressure, $C V P$ central venous pressure, $\mathrm{PaCO}_{2}$ arterial $\mathrm{CO}_{2}$ pressure, $\mathrm{PaO}_{2}$ arterial oxygen pressure, $\mathrm{SaO}_{2}$ oxygen saturation, $I C U$ intensive care unit, ESR erythrocyte sedimentation rate, $L D H$ lactate dehydrogenase, $H b$ hemoglobin, $A L A T$ alanine aminotransferase, ASAT aspartate aminotransferase, $A K I$ acute kidney injury. ${ }^{\text {Significance level }}$ presented is for AKI 1-3 combined, given that there are no patients in the mitral + coronary group with AKI 3.
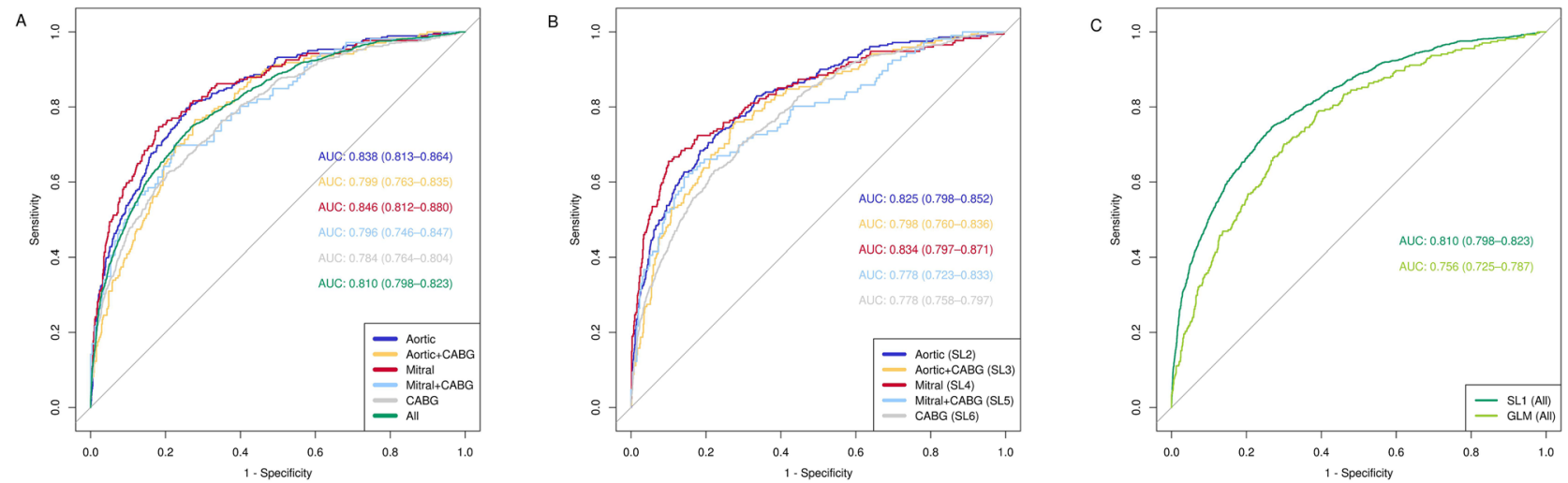

Figure 1. Plot of the receiver operating characteristic (ROC) curves and the respective areas under curve (AUCs) for the weighted Super Learner 1 for each of the 5 types of operation and for the whole cohort. Plot of the receiver operating characteristic (ROC) curves and the respective areas under curve (AUCs) for the weighted Super Learner and the generalized linear model (GLM) for the whole cohort. SL super learner, $C A B G$ coronary artery bypass grafting.

to baseline. Likewise, mortality risk gradually increased between 60 and 80 years of age. Figure 4 illustrates the combined effect of serum urea day 4 and age on mortality risk.

\section{Discussion}

This study shows that ensemble ML analysis achieves a high accuracy in predicting 5-year mortality in a cohort of 8241 patients with CABG and/or valve operations. Moreover, variable importance analysis revealed early postoperative urea as a novel and strong predictor of mortality in all types of cardiac operations. Furthermore, methodologically, a more targeted approach of training the algorithms on sub-groups instead of the full cohort did not significantly improve mortality prediction.

We demonstrated that using an ensemble algorithm with a combination of pre-operative, intra-operative, and first week post-operative data, achieves high accuracy in predicting 5-year mortality after different types of cardiac operations. These findings extend a previous study where we demonstrated the superiority of individual ML models compared to classical multivariable analysis in identifying patients at increased risk of long-term mortality after $\mathrm{CABG}^{7}$. Here, we reaffirm these findings using ensemble $\mathrm{ML}$ and data from different types of cardiac operations. Using peri-operative data, we achieved similar accuracy to a recently developed ML-based risk algorithm for prediction of 1- to 24-month mortality following major surgery ${ }^{15}$. Compared to other models that predict mortality specifically after cardiac surgery, the ensemble achieved superior performance ${ }^{8}$.

The application of algorithms such as the one we developed to pre-operative data would possibly predict patients at the highest risk of long-term complications prior to surgery. Expectedly, analysis of pre-operative data in the XGBoost model decreased performance significantly, which could be partly due to the limited set of pre-operative data available in our cohort, or to the lower frequency of the outcome (long-term mortality 


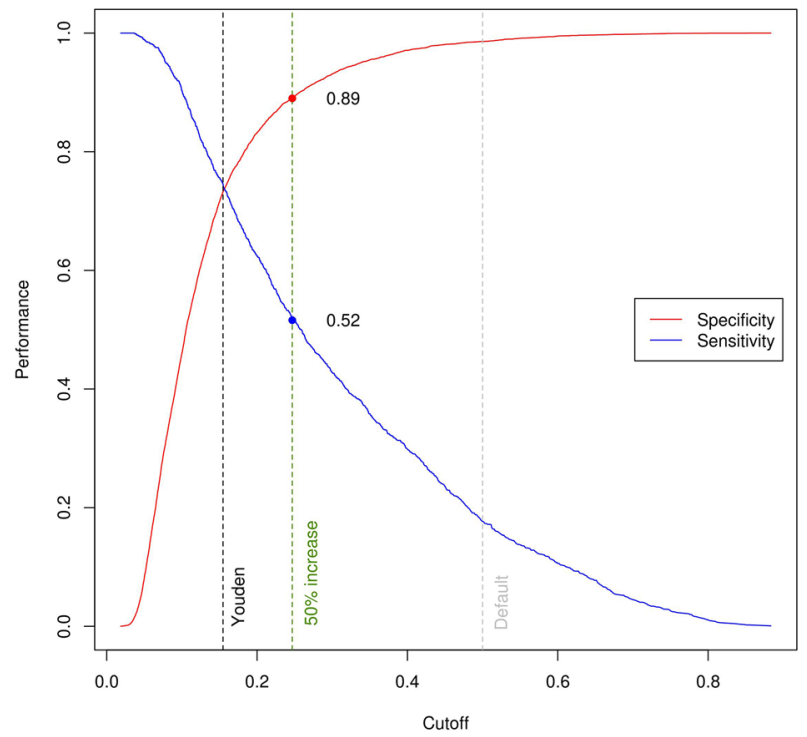

Figure 2. Specificity (blue) and sensitivity (red) values across all possible thresholds for all operations combined. The default 0.50 threshold is marked in grey, the threshold based on the maximized Youden index in black, and the threshold representing a 50\% increase in mortality risk in green.
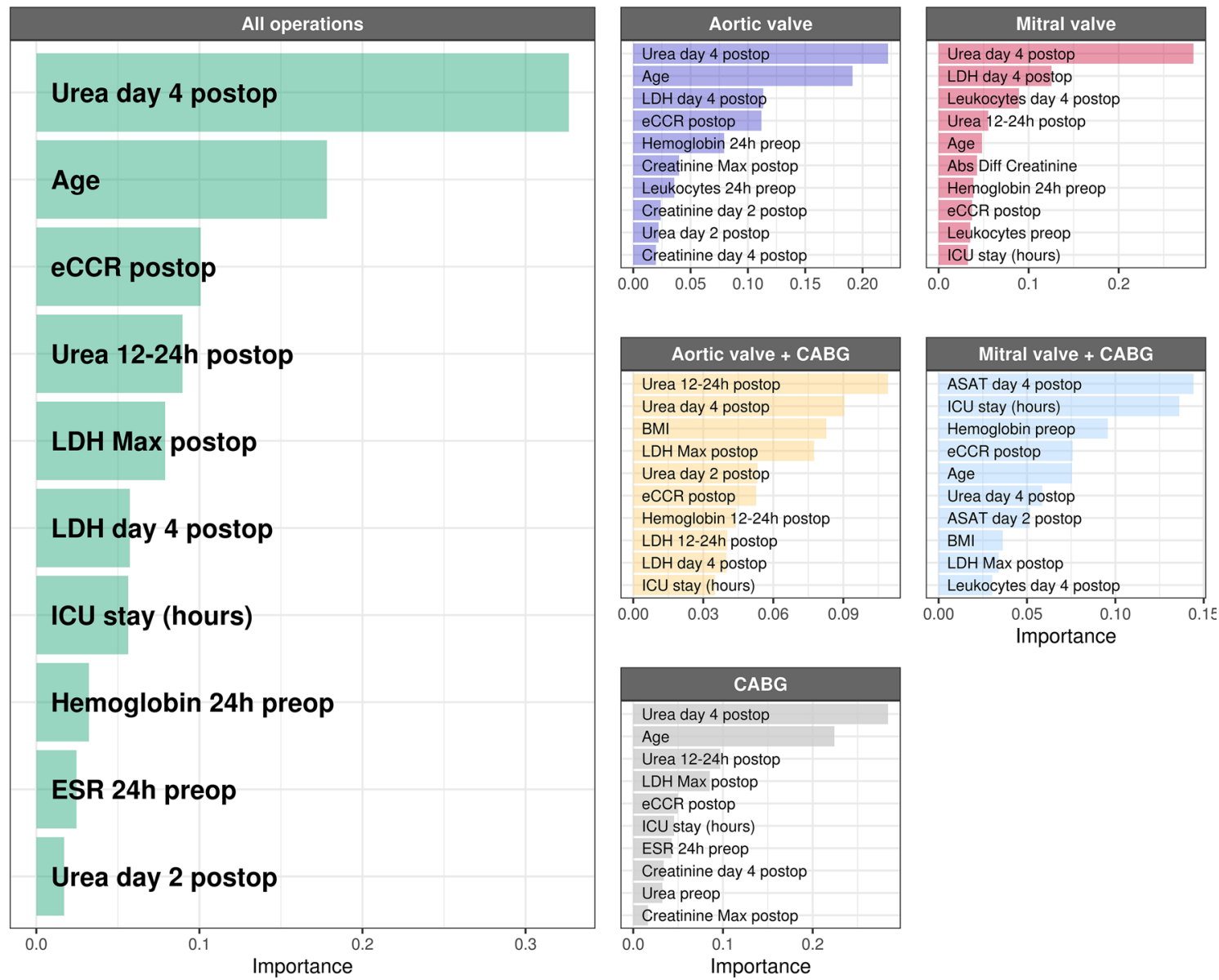

Figure 3. Top ten predictor variables for all types of operations combined. Variable coefficients indicate how much each parameter contributes to the outcome. $e C C R$ estimated creatinine clearance, $L D H$ lactate dehydrogenase, ESR erythrocyte sedimentation rate, ICU intensive care unit, ASAT aspartate transaminase, BMI body mass index. 


\begin{tabular}{|c|c|c|}
\hline & \multicolumn{2}{|c|}{$\begin{array}{l}\text { Predictions matching actual patient } \\
\text { outcome }(\%)\end{array}$} \\
\hline & Survivors (\%) & Non-survivors (\%) \\
\hline \multicolumn{3}{|l|}{ Aortic valve } \\
\hline With default threshold & 98.8 & 18.1 \\
\hline With $50 \%$ increased risk threshold & 90.5 & 53.0 \\
\hline Difference & -8.3 & +34.9 \\
\hline \multicolumn{3}{|l|}{ Mitral valve } \\
\hline With default threshold & 96.8 & 34.5 \\
\hline With 50\% increased risk threshold & 89.7 & 59.8 \\
\hline Difference & -7.1 & +25.3 \\
\hline \multicolumn{3}{|l|}{ CABG } \\
\hline With default threshold & 99.2 & 10.4 \\
\hline With $50 \%$ increased risk threshold & 88.9 & 47.9 \\
\hline Difference & -9.3 & +37.5 \\
\hline \multicolumn{3}{|l|}{ Aortic + CABG } \\
\hline With default threshold & 97.0 & 19.9 \\
\hline With 50\% increased risk threshold & 88.8 & 43.3 \\
\hline Difference & -8.2 & +23.4 \\
\hline \multicolumn{3}{|l|}{ Mitral + CABG } \\
\hline With default threshold & 96.9 & 28.3 \\
\hline With $50 \%$ increased risk threshold & 95.4 & 34.9 \\
\hline Difference & -1.5 & +6.6 \\
\hline \multicolumn{3}{|l|}{ All operations combined } \\
\hline With default threshold & 98.6 & 17.7 \\
\hline With $50 \%$ increased risk threshold & 89.4 & 51.6 \\
\hline Difference & -9.2 & +33.9 \\
\hline
\end{tabular}

Table 2. Percentage of correctly classified cases in survivors and non-survivors per operation type for SL1 predictions using the default and 50\% increase in risk thresholds.

as opposed to short-term post-operative complications). Yet, it should be noted that the model's performance using our restricted set of pre-operative data has comparable predictive power as currently used clinical scores ${ }^{8}$.

Methodologically, our study contributed to the discussion on the need of conducting predictive studies on operation-specific cohorts. Results from previous studies suggest that algorithms trained on pooled data from patients undergoing different types of surgeries were accurate in predicting outcomes for all these types of operations. In keeping, our findings show that both the model trained with the full cohort, and the models trained with the individual cardiac operation subgroups showed a good performance in predicting long-term mortality after aortic and mitral valve operations. This finding further questions the need to conduct ML analyses on operation-specific cohorts. Specifically, including full cohorts may lead to better model performance analyses due to the greater amount of data.

Additionally, by providing risk predictions at individual level, ML algorithms allow for the adjustment of the sensitivity and specificity of each model for different clinical settings ${ }^{15}$. Balancing sensitivity and specificity in the context of mortality risk predictions can be challenging. Lowering the prediction threshold may lead to excessive over-diagnosing and increase in healthcare costs. However, especially in populations with relatively low mortality rates such as cardiac surgery patients, a too high threshold would miss too many "non-survivors". Here, we demonstrated that using a $50 \%$ increase in absolute risk of mortality as cut-off provides a favorable trade-off between false positives and true negatives, as previously shown in similar large studies predicting postoperative mortality and mortality in intensive care patients ${ }^{15,16}$. Validation of this approach merits further investigation, and may facilitate the translation of an algorithm's good predictive performance into a clinically useful patient risk stratification tool ${ }^{17}$.

Variable importance analysis identified postoperative urea as the strongest predictor of 5-year mortality. This is consistent with our previous findings in a CABG-only population ${ }^{7}$. Yet, literature on the possible role of urea as a mortality predictor in cardiac operations is scarce ${ }^{7}$. Preoperative urea values above $10 \mathrm{mmol} / \mathrm{L}$ have been found to be associated with increased 30-day mortality risk after CABG and with increased risk of stroke in the 10 days after cardiac operations ${ }^{18,19}$. It should also be noted that, in heart failure patients, increased urea levels have been associated with derangements in cardiac output and renal perfusion ${ }^{20,21}$. These are, in turn, strongly related to patients' overall performance status and prognosis, with both urea and the urea/creatinine ratio being known prognostic predictors ${ }^{22}$. In the context of this study, increased urea may originate from excess production and/or impaired excretion, yet mechanistic insight remains elusive. Possibly, urea production may be increased by mitochondrial dysfunction, caused by ischemia/reperfusion and increased systemic inflammatory response after cardiopulmonary bypass and surgical trauma ${ }^{23}$. Mitochondrial dysfunction may be amplified through excess 

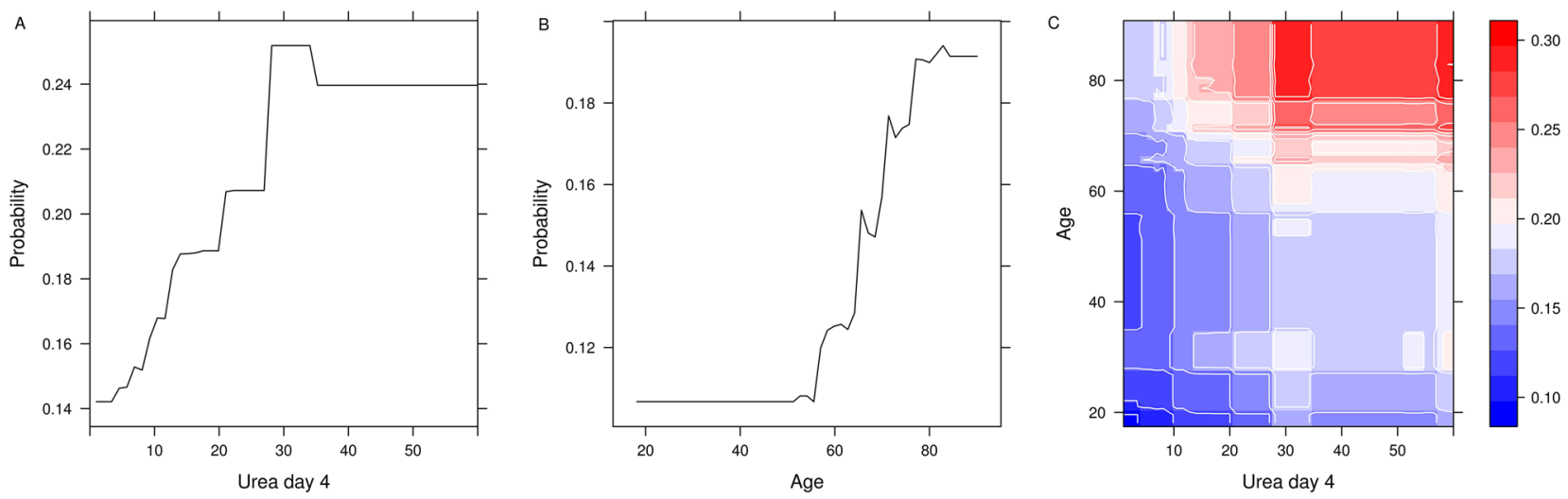

Figure 4. Partial dependence plots of urea at postoperative day 4 and age. Partial dependence plots of urea at postoperative day 4 against age. The vertical bar represents predicted risk (blue to red, low to high).

reactive oxygen species (ROS) following accumulation of succinate during ischemia ${ }^{24,25}$. Additionally, recent evidence indicates that high urea levels generate $\operatorname{ROS}^{26}$. Furthermore, renal excretion of urea may decrease in response to kidney injury. Thus, urea likely reflects the compound pathological state of different organ systems, rather than just kidney function.

Lastly, this study also has some limitations to consider. Being a single center study, our findings need confirmation by external validation. Further, our analysis is limited to the variables in the CAROLA database. Detailed co-morbidity information, for instance, could help further improve model performance, especially for the CABG sub-group. Additionally, variable importance analysis as such does not provide directionality and assumptions about effect size between the variables and the outcome cannot be made directly. Finally, the current ensemble ML is not suited to use high-frequency, high-volume data, such as continuous intraoperative measurements of blood pressure, heart rate, oxygen saturation or temperature. Therefore, a study including algorithms suitable for such analysis, such as recurrent neural networks, is a logical follow-up.

In conclusion, ML analysis of 88 routinely collected peri-operative data achieved a high accuracy in predicting 5-year mortality after different cardiac operations in this large study of 8241 patients. A targeted approach of training the algorithms on sub-groups instead of the full cohort did not improve model performance. Moreover, variable importance analysis showed early postoperative urea as a novel and strong predictor of mortality in all types of cardiac operations. Similar studies enabling the identification of modifiable risk factors and providing individual patient predictions may form a first step towards facilitating personalized clinical interventions to improve patient care.

\section{Methods}

The electronic Cardiothoracic Anesthesiology Registry (CAROLA) comprises extensive prospective data of all adult patients who underwent first-time valve operation, CABG, or a combination of both between 1997 and 2017 in the University Medical Centre Groningen (UMCG), the Netherlands. The total number of patients is 11,286 . This database study was approved by the Medical Ethical Committee of the UMCG, and the requirement to obtain informed consent was waived (waiver: METC\#2010/118). All analyses were performed in accordance with relevant guidelines and regulations.

Patient population and outcome. Only patients who underwent valve operation, either solitary or combined with coronary artery bypass grafting (CABG), or solitary CABG, with cardiopulmonary bypass (CPB) were included $(n=8241)$. There were 1663 patients in the combined aortic and coronary group, 367 in the combined mitral and coronary group, 884 in the solitary mitral group, 813 in the solitary aortic group, and 4514 in the CABG-only group. Mortality data were obtained in November 2017 from the Dutch Municipal Personal Records Database comprising actual and reliable data of all citizens within the Netherlands.

Data selection and pre-processing. The dataset includes patient characteristics, peri-operative hemodynamic, $\mathrm{CPB}$, respiratory and organ function data and blood values collected at different time points indicated in Fig. 5. Because for some patients referred from other hospitals the stay in our center was limited to the immediate peri-operative phase, a variable pattern of missing data was observed. Multivariate imputation by chained equations was performed on the set of variables with at least $50 \%$ non-missing data ${ }^{27}$. The final dataset without missing data consisted of 88 predictor variables and 5-year mortality as the outcome variable (Table 1). Baseline serum creatinine measurements was defined as the closest to the start of operation. Patients were classified for post-operative AKI 0-3 within the 7 days after operation according to the AKIN classification ${ }^{3}$.

Statistical analysis. The Super Learner, selected candidate algorithms, and hyper-parameter tuning. The Super Learner algorithm is a generalization of the stacking algorithms developed by Breiman ${ }^{28}$, which combines a set of candidate algorithms to make k-fold-cross-validated predictions ${ }^{9,29}$. In this process, the dataset is divided into $\mathrm{k}$ mutually exclusive and exhaustive subsets, with one set serving as a validation set, while the others are 


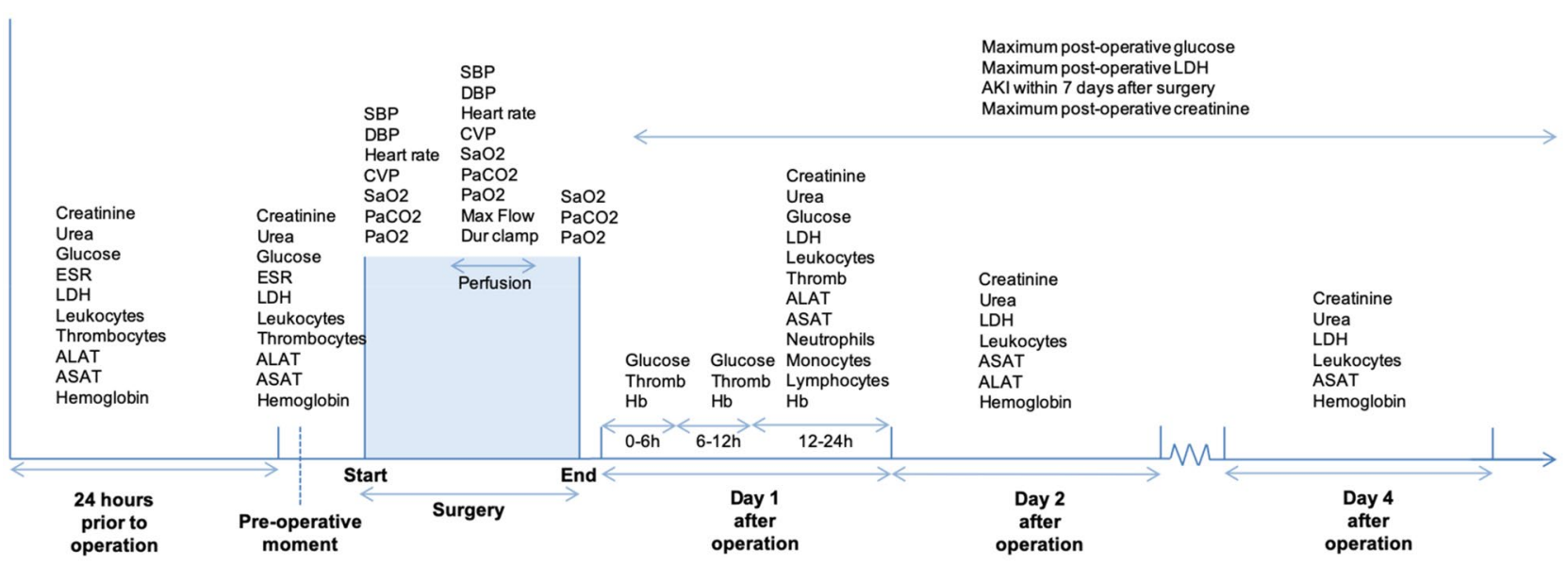

Figure 5. Timeline of clinical measurements before, during, and after cardiac operation, in the intensive care unit (day 1 after operation), day 1 in the ward (day 2 after operation), and day 3 in the ward (day 4 after operation). Patient characteristics are not included here, but described in detail in Table 1. Dur CA duration of cardiac arrest, Dur clamp duration of aortic cross-clamp, $H b$ hemoglobin, ASAT aspartate aminotransferase, $A L A T$ alanine aminotransferase, Thromb thrombocytes, ESR erythrocyte sedimentation rate, $L D H$ lactate dehydrogenase, $\mathrm{CVP}$ central venous pressure, $\mathrm{PaCO}_{2}$ arterial carbon dioxide partial pressure, $\mathrm{SaO}_{2}$ oxygen saturation, $\mathrm{PaO}_{2}$ arterial oxygen partial pressure, $S B P$ systolic blood pressure, $D B P$ diastolic blood pressure.

used for training each candidate algorithm ${ }^{14}$. This means that each patient is used only once in the validation set, and included in the training set for all other rounds. For each candidate learner, $\mathrm{k}$ risks are calculated and averaged into a "cross-validated risk". Subsequently, the learners with the minimal risk are selected, applied to the entire dataset and included in the new weighted estimator (the SL), that attributes a relative coefficient to each of the learners. Those which reduce the calculated risk the most, will contribute to the final weighted prediction. Moreover, the SL presents individual patient predicted probabilities for 5-year mortality per ensemble. Five candidate algorithms were included in the SL: support Bayesian additive regression trees (BART), extremely randomized trees, elastic net, support vector machine, and extreme gradient boosted machine (XGBoost). Details of these five algorithms can be found in the "Supplementary material". Since the performance of an algorithm varies greatly depending on its hyper-parameters and can be substantially improved by tuning, multiple hyper-parameter combinations were generated for each candidate algorithm. Details of each of these algorithms including the hyper-parameters, the tuning process, and final values are described in the "Supplementary material". A 10-fold cross-validated generalized linear regression model (GLM) was trained on data from the full cohort for use as baseline comparison of the SL's performance. Lastly, to test the performance of a model using only pre-operative data in predicting post-operative outcomes, a 10-fold cross-validated XGBoost model was trained on data from the full cohort.

Model training. Two distinct training procedures for the SL were carried out (Fig. 6). First, one of the ensembles (SL1) was trained using the full cohort of 8241 patients. Secondly, the cohort was split into five different groups according to operation type, with one ensemble trained on data from each group (SL2-SL6). All six ensembles included the same candidate algorithms, and the same hyper-parameter configurations. Performance of two different approaches were assessed by comparison of the 10-fold cross-validated area under the receiver operated characteristic curve (AUROC), with a 95\% confidence interval, for each of the weighted SL's. Differences in the performance between SL's and between SL1 and the GLM were assessed with DeLong's nonparametric test for the difference in areas under the curve ${ }^{30}$.

Calibration, sensitivity analysis and adjusted risk thresholds based on predicted probability of mortality. Calibration plots and calibration indices (ECI) $)^{31}$ for all models are provided in the "Supplementary material". Model performance metrics described above were obtained in a 2-step procedure: first using a default threshold to maximize the AUROC, and then using adjusted thresholds to optimize sensitivity and specificity. This process of tuning the operating points of the ROC using different risk thresholds depending on the requirements of a specific clinical setting has been previously shown to optimize model sensitivity and specificity for mortality prediction ${ }^{15}$. In the first step, a default threshold of 0.50 was used, where patients are classified as "non-survivors" if the predicted probability of mortality is greater than 50\%. This is the standard threshold used to maximize algorithm performance during training. After this, a second and third risk thresholds were defined. The second one was calculated based on the maximized Youden index, which provides a balance between sensitivity and specificity ${ }^{15}$. The third one was based on the actual long-term mortality rate of each of the surgical sub-groups, and corresponds to a $50 \%$ increase in the absolute risk of mortality. We opted for this value as it represents a clinically relevant increase that could justify intervention. The confusion matrix, sensitivity, and specificity for each of the thresholds are reported in the "Supplementary material". 


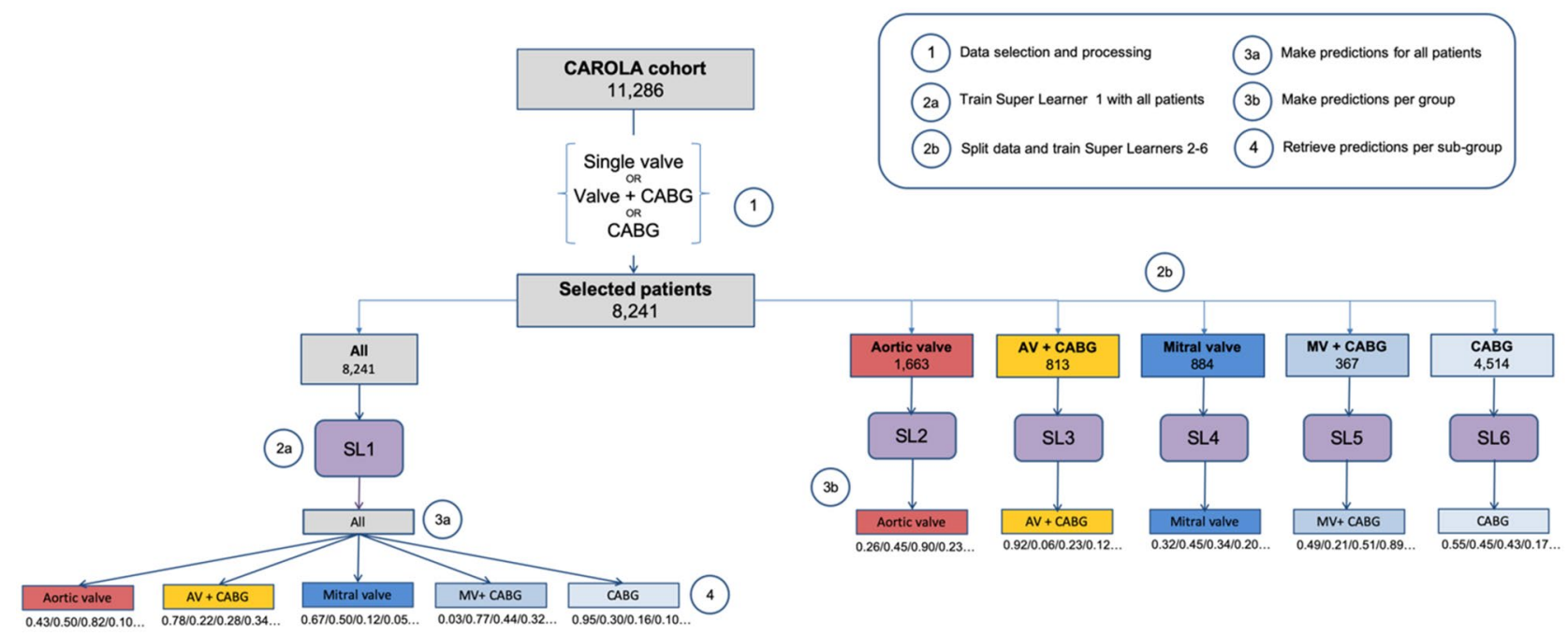

Figure 6. Diagram of the steps involved in data analysis: data split, algorithm training, and outcome prediction using different Super Learner ensembles. On the left, the process of training the single Super Learner on data of the whole cohort $(n=8241)$, obtaining the pooled predicted probabilities, and retrieving the group-specific probabilities to calculate the performance measures for each type of operation. On the right, the process of splitting the data into five groups, one per operation type, and training a different super learner on data from one type of operation only. $S L$ super learner, $A V$ aortic valve, $M V$ mitral valve, $C A B G$ coronary artery bypass grafting.

Variable importance analysis. Variable importance measures aim at estimating the contribution of predictor variables to changes in the outcome ${ }^{32}$. The greater the association between each feature and the outcome, the greater the decrease in accuracy upon its removal, and the higher its reported importance ${ }^{32}$. We determined the variable importance of all routinely measured peri-operative clinical parameters in our cohort by training the best performing individual algorithm included in the ensemble — the XGBoost model—using the same hyperparameter configurations as in the SL. The coefficients for the top ten features for each operation type, as well as for all operations combined, are presented.

All analyses were performed using R version 3.6.2 (The R Foundation for Statistical Computing; Vienna, Austria) for Ubuntu 16.04 LTS. Data are expressed as mean (95\% confidence interval), and categorical as percentages. A $P$ value $<0.05$ was accepted as a statistically significant difference.

Received: 27 June 2020; Accepted: 20 January 2021

Published online: 10 February 2021

\section{References}

1. Loef, B. G. et al. Immediate postoperative renal function deterioration in cardiac surgical patients predicts in-hospital mortality and long-term survival. J. Am. Soc. Nephrol. 16(1), 195-200 (2005).

2. Loef, B. G., Epema, A. H., Navis, G., Ebels, T. \& Stegeman, C. A. Postoperative renal dysfunction and preoperative left ventricular dysfunction predispose patients to increased long-term mortality after coronary artery bypass graft surgery. Br. J. Anaesth. 102(6), 749-755 (2009).

3. Mehta, R. L. et al. Acute kidney injury network: Report of an initiative to improve outcomes in acute kidney injury. Crit. Care. 11(2), R31 (2007).

4. Lassnigg, A. et al. Minimal changes of serum creatinine predict prognosis in patients after cardiothoracic surgery: A prospective cohort study. J. Am. Soc. Nephrol. 15(6), 1597-1605 (2004).

5. Bouma, H. R. et al. Acute kidney injury classification underestimates long-term mortality after cardiac valve operations. Ann. Thorac. Surg 106(1), 92-98 (2018).

6. D'Agostino, R. S. et al. The Society of Thoracic Surgeons Adult Cardiac Surgery Database: 2018 update on outcomes and quality. Ann. Thorac. Surg. 105, 15-23 (2018).

7. Forte, J. N. C., Wiering, M. A., Bouma, H. R., de Geus, A. G. \& Epema, A. H. Predicting long-term mortality with first week postoperative data after Coronary Artery Bypass Grafting using Machine Learning models. PMLR 68, 39-58 (2017).

8. Allyn, J. et al. A comparison of a machine learning model with euroscore II in predicting mortality after elective cardiac surgery: A decision curve analysis. PLoS ONE 12(1), e0169772 (2017).

9. Pirracchio, R. et al. Mortality prediction in intensive care units with the Super ICU Learner Algorithm (SICULA): A populationbased study. Lancet Respir. Med. 3(1), 42-52 (2015).

10. Nanayakkara, S. et al. Characterising risk of in-hospital mortality following cardiac arrest using machine learning: A retrospective international registry study. PLoS Med. 15(11), e1002709 (2018).

11. Cherifa, M. et al. Prediction of an acute hypotensive episode during an ICU hospitalization with a super learner machine-learning algorithm. Anesth. Analg. 130(5), 1157-1166 (2020).

12. Hatib, F. et al. Machine-learning algorithm to predict hypotension based on high-fidelity arterial pressure waveform analysis. Anesthesiology 129, 663-674 (2018).

13. Davies, S. J., Vistisen, S. T., Jian, Z., Hatib, F. \& Scheeren, T. W. L. Ability of an arterial waveform analysis-derived hypotension prediction index to predict future hypotensive events in surgical patients. Anesth. Analg. 130, 352-359 (2020).

14. van der Laan, M. J., Polley, E. C. \& Hubbard, A. E. Super learner. Stat. Appl. Genet. Mol. Biol. 6(1), 1544-6115 (2007). 
15. Bihorac, A. et al. MySurgeryRisk: Development and validation of a machine-learning risk algorithm for major complications and death after surgery. Ann. Surg. 269(4), 652-662 (2019).

16. Thorsen-Meyer, H.-C. et al. Dynamic and explainable machine learning prediction of mortality in patients in the intensive care unit: A retrospective study of high-frequency data in electronic patient records. Lancet Digital Health. 2(4), e179-e191 (2020).

17. Gordon, L., Austin, P., Rudzicz, F. \& Grantcharov, T. MySurgeryRisk and machine learning: A promising start to real-time clinical decision support. Ann. Surg. 269(1), e14-e15 (2019).

18. Arnan, M. K. et al. Postoperative blood urea nitrogen is associated with stroke in cardiac surgical patients. Ann. Thorac. Surg. 99, 1314-1320 (2015).

19. Chung, P. J. et al. Predicting the risk of death following coronary artery bypass graft made simple: a retrospective study using the American College of Surgeons National Surgical Quality Improvement Program database. J. Cardiothorac. Surg. 10, 62 (2015).

20. Kazory, A. Emergence of blood urea nitrogen as a biomarker of neurohormonal activation in heart failure. Am. J. Cardiol. 106, 694-700 (2010).

21. Gotsman, E. et al. The significance of serum urea and renal function in patients with heart failure. Medicine. 89(4), 197-203 (2010).

22. Matsue, Y. et al. Blood urea nitrogen-to-creatinine ratio in the general population and in patients with acute heart failure. Heart 103(6), 407-413 (2017).

23. Cherry, A. D. Mitochondrial dysfunction in cardiac surgery. Anesthesiol. Clin. 37(4), 769-785 (2019).

24. Chouchani, E. T. et al. Ischaemic accumulation of succinate controls reperfusion injury through mitochondrial ROS. Nature 515, 431-435 (2014).

25. Sun, J. et al. Mitochondria in sepsis-induced AKI. J. Am. Soc. Nephrol. 30(7), 1151-1161 (2019).

26. D'Apolito, M. et al. Urea-induced ROS cause endothelial dysfunction in chronic renal failure. Atherosclerosis. 239(2), 393-400 (2015).

27. van Buuren, S. \& Groothuis-Oudshoorn, K. Mice: Multivariate imputation by chained equations in r. J. Stat. Softw. 45(3), 1-67 (2011).

28. Breiman, L. Bagging predictors. Mach. Learn. 24, 123-140 (1996).

29. Dudoit, S., van der Laan, M.J. Asymptotics of cross-validated risk estimation in estimator selection and performance assessment. Accessed 1 June 2020; http://biostats.bepress.com/ucbbiostat/paper126/. (2006).

30. DeLong, E. R., DeLong, D. M. \& Clarke-Pearson, D. L. Comparing the areas under two or more correlated receiver operating characteristic curves: A nonparametric approach. Biometrics 44(3), 837-845 (1988).

31. van Hoorde, K., Van Huffel, S., Timmerman, D., Bourne, T. \& Van Calster, B. A spline-based tool to assess and visualize the calibration of multiclass risk predictions. J. Biomed. Inform. 54, 283-293 (2015).

32. Díaz, I., Hubbard, A., Decker, A. \& Cohen, M. Variable importance and prediction methods for longitudinal problems with missing variables. PLoS ONE 10(3), e0120031 (2015).

\section{Author contributions}

J.C.F., M.W., R.H., and A.E. designed and directed the study. J.C.F., M.W., and M.G. selected and implemented the machine learning algorithms. J.C.F., V.P., I.H., R.H., and A.E. drafted the paper. H.M., F.G., H.B., T.W.L.S., M.N., and M.M. contributed to data acquisition and revised the paper. All authors read and approved the manuscript.

\section{Competing interests}

TWLS received research grants and honoraria from Edwards Lifesciences (Irvine, CA, USA) and Masimo Inc. (Irvine, CA, USA) for consulting and lecturing and from Pulsion Medical Systems SE (Feldkirchen, Germany) for lecturing. All other authors have no competing interests to report.

\section{Additional information}

Supplementary Information The online version contains supplementary material available at https://doi. org/10.1038/s41598-021-82403-0.

Correspondence and requests for materials should be addressed to J.C.F.

Reprints and permissions information is available at www.nature.com/reprints.

Publisher's note Springer Nature remains neutral with regard to jurisdictional claims in published maps and institutional affiliations.

Open Access This article is licensed under a Creative Commons Attribution 4.0 International License, which permits use, sharing, adaptation, distribution and reproduction in any medium or format, as long as you give appropriate credit to the original author(s) and the source, provide a link to the Creative Commons licence, and indicate if changes were made. The images or other third party material in this article are included in the article's Creative Commons licence, unless indicated otherwise in a credit line to the material. If material is not included in the article's Creative Commons licence and your intended use is not permitted by statutory regulation or exceeds the permitted use, you will need to obtain permission directly from the copyright holder. To view a copy of this licence, visit http://creativecommons.org/licenses/by/4.0/.

(C) The Author(s) 2021 